\title{
Ausgeprägte Akne vulgaris bei eineiigen Zwillingen - eine besondere Herausforderung
}

\author{
Serious Acne Vulgaris in Monozygotic Twins - A Particular Challenge
}

Autor G. Reimer

Bibliografie

DOI $10.1055 / \mathrm{s}-2006-944582$

Akt Dermatol 2006; 32;

425-428 @ Georg Thieme

Verlag KG Stuttgart · New York ISSN 0340-2541

Korrespondenzadresse Dr. Dr. med. Günter Reimer Kaiser-Friedrich-Promenade 6 61348 Bad Homburg v. d. Höhe info@hautarzt-dr-reimer.de

\section{Zusammenfassung $\nabla$}

Nach einer Reihe von weniger erfolgreichen Therapieversuchen stellten sich zwei 18-jährige Zwillingsschwestern mit einer ausgeprägten Akne papulopustulosa vor. Schweregrad und Lokalisation waren sehr ähnlich, wie dies für eineiige Zwillinge zu erwarten ist. Da die Patientinnen eine orale Isotretinoin-Behandlung ablehnten, wurde zunächst mit systemischem Antibiotikum in Kombination mit einer Tretinoin/Erythromycin-Creme behandelt. Zusatzleistungen gewährleisteten eine intensive Patientenführung. Wegen nicht zufrieden stellender Resultate wurde zunächst eine der Zwillingsschwestern auf ein neu-

\section{Anamnese}

$\nabla$

Die eineiigen Zwillingsschwestern Franziska und Stefanie leiden seit dem 11. Lebensjahr unter Akne. Zur Reihe der Vorbehandlungen gehörten topische Therapeutika (Erythromycin/Zinkacetat, Natriumbituminosulfonat) sowie systemisches Minocylin über einen Zeitraum von 12 Monaten. Die Schwestern haben sehr ähnliche Lebensgewohnheiten und wurden stets analog therapiert. Aufgrund wenig zufrieden stellender Resultate vorangegangener Behandlungen und drohender Narbenbildung wurde den Patientinnen eine systemische Isotretinoin-Therapie nahe gelegt. Diese lehnten beide Schwestern aufgrund von Bedenken wegen möglicher Nebenwirkungen ebenso ab wie die erforderliche Kontrazeption.

Daher wurden die Patientinnen zunächst auf orales Doxycyclin (Dosierung: $2 \times \mathrm{tgl} .100 \mathrm{mg}$ ) eingestellt. Dieses wurde mit einer topischen Tretinoin/Erythromycin-Creme (0,2-proz. VitaminA-Säure in 2-proz. hydrophiler ErythromycinCreme NRF 11.77, einmal täglich, abends) kombiniert. es topisches Kombinationspräparat mit $1 \%$ Clindamycin und 5\% Benzoylperoxid (Duac ${ }^{\circledR}$ Akne Gel) umgestellt. Aufgrund der bereits nach 3 Wochen sichtbaren Verbesserung bei dieser Patientin wurde von diesem Zeitpunkt an auch ihre Schwester ausschließlich mit diesem Präparat weiter behandelt. In beiden Fällen kam es zu einem weitestgehenden Rückgang der entzündlichen Effloreszenzen, wobei für eine gewisse Zeitdauer der zeitlich verzögerte Therapiewechsel sichtbar blieb. Im Vergleich zur Therapie mit oralem Antibiotikum plus Tretinoin/ErythromycinCreme erwies sich die alleinige Behandlung mit Duac ${ }^{\circledR}$ Akne Gel letztlich als deutlich effektiver.

Zur adjuvanten Hautpflege verwendeten beide Patientinnen während des gesamten Behandlungszeitraumes morgens eine Ammoniumlactat-haltige Pflegecreme.

Begleitend zur pharmakologischen Therapie erhielten die Zwillinge alle 4 Wochen eine Behandlung mit einer IPL (Intense Pulsed Light)-Blitzlampe (DEKA, Typ Photosilk plus bei Wellenlängen von 550-900 nm, mit Trippel-Puls je $3 \mathrm{~ms}$ und einer Gesamt-Energiedichte von $11.0 \mathrm{~J} / \mathrm{cm}^{2}$ je Behandlung). Durch diese schmerzfrei verlaufende Photothermolyse der Talgdrüsen-Kapillaren konnte die Sebumproduktion verringert werden. Ebenfalls im 4-Wochen-Rhythmus, nur um 14 Tage verschoben, erfolgte ein Fruchtsäurepeeling [70-proz. Ammoniumlactat-Gel mit pH 2.1], so dass sich die Patientinnen 14-tägig in der Praxis vorstellten, was eine intensive Patientenführung gewährleistete.

Da sich auch nach drei Monaten bei keiner der Patientinnen eine hinreichende Besserung eingestellt hatte ( $\bullet$ Abb. 1), diese aber die anderen systemischen Therapieoptionen weiterhin ablehnten, wurde zunächst das Antibiotikum gewechselt. Anstelle des Tetracyclin-Derivates Dox- 


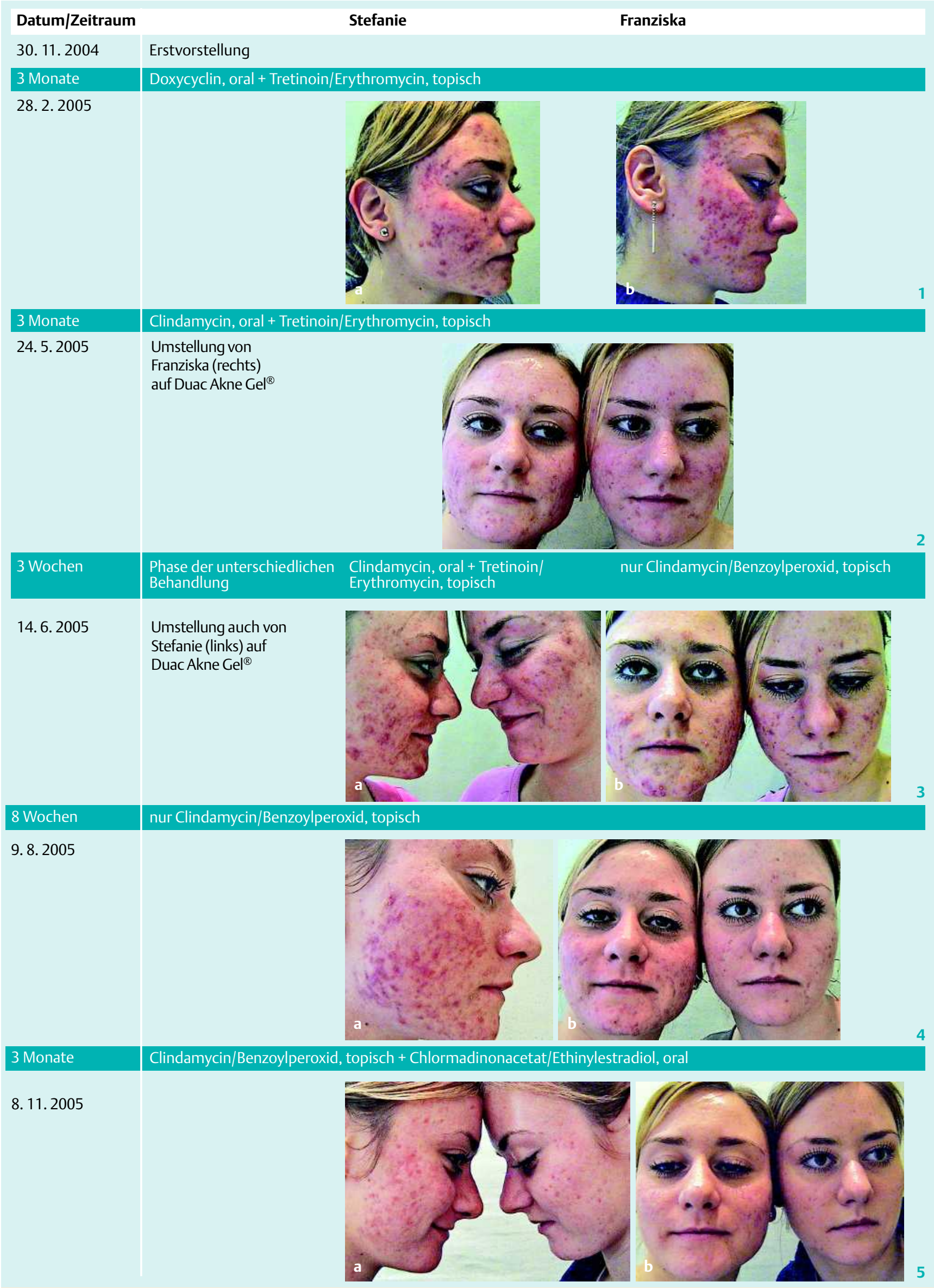

Abb. 1-5 Dokumentation des Therapieverlaufs mit seinen verschiedenen Phasen. 
ycyclin erhielten die Zwillinge nun das Lincosamid-Antibiotikum Clindamycin (Dosierung: $2 \times$ tgl. $300 \mathrm{mg}$ oral). Die abendliche Applikation der VAS/Erythromycin-Creme wurde beibehalten.

\section{Aufnahmebefund \\ $\nabla$}

Die eineiigen Zwillingsschwestern Franziska und Stefanie stellten sich gemeinsam im Alter von 18 Jahren mit einer ausgeprägten Akne papulopustulosa im Gesicht vor. Schweregrad und Lokalisation waren sehr ähnlich, wie es bei eineiigen Zwillingen aufgrund weitgehend übereinstimmender Sebumexkretionsraten zu erwarten ist [7].

Unter dem zuletzt applizierten systemischen Clindamycin kam es innerhalb von 3 Monaten bei beiden zu einer sichtbaren, aber weiterhin nicht zufrieden stellenden Besserung des Befundes ( $\odot$ Abb. 2). Zu berücksichtigen war dabei eine erhöhte psychische Belastung der Zwillingsschwestern durch deren intensiven (Blick-)Kontakt untereinander: Beide hatten gewissermaßen regelmäßig ihr Spiegelbild vor Augen. Damit ergab sich trotz der umfangreichen therapeutischen und therapiebegleitenden Maßnahmen sowie der intensiven Kommunikation die Gefahr einer Verschlechterung der Compliance.

\section{Therapie und Verlauf}

$\checkmark$

Im Mai 2005 wurde gerade ein neues topisches Aknemittel mit $1 \%$ Clindamycin und 5\% Benzoylperoxid (Duac ${ }^{\circledR}$ Akne Gel) in Deutschland eingeführt. Dieses Kombinationspräparat hatte sich in klinischen Studien [3,5] den Einzelkomponenten als signifikant überlegen erwiesen und zeichnete sich nach weiteren Vergleichsstudien mit anderen bewährten Lokaltherapeutika $[1,2]$ vor allem durch seinen schnellen Wirkungseintritt aus. Des Weiteren bewirkte die Kombination Clindamycin/Benzoylperoxid bei In-vitro-Untersuchungen [4] eine stärkere Reduktion von Propionibacterium acnes als verschiedene andere topische und auch orale Antibiotika. In einer randomisierten, kontrollierten klinischen Studie [6] wurde weiterhin gezeigt, dass eine Kombination von Benzoylperoxid mit topischem Antibiotikum im Vergleich von fünf antimikrobiellen Therapieregimen tendenziell zum deutlichsten Rückgang der entzündlichen Effloreszenzen führte. Der prozentuale Anteil der Patienten mit mindestens moderater Besserung war nach 18 Wochen bei der topischen Kombinationstherapie höher als im Fall von systemisch appliziertem Minocyclin.

Aufgrund der überzeugenden Datenlage und der fehlenden Zustimmung für eine orale Isotretinoin-Behandlung entschloss sich der behandelnde Arzt, bei einer der Schwestern auf das systemische Antibiotikum und die Tretinoin/Erythromycin-Creme zu verzichten und auf eine alleinige Behandlung mit Duac ${ }^{\circledR}$ Akne Gel umzustellen. Während Franziska nunmehr einmal abends Duac ${ }^{\circledR}$ Akne Gel auftrug, führte Stefanie die Behandlung mit Clindamycin oral und Tretinoin/Erythromycin fort. Die Zwillinge waren mit dieser erstmals unterschiedlichen Behandlung zunächst einverstanden, da auch sie in Erfahrung bringen wollten, ob das neue Präparat ihnen wirklich besser helfen könne als die bisherige Therapie.

Bereits 3 Wochen nach der Umstellung war bei den Schwestern ein deutlicher Unterschied im klinischen Befund festzustellen ( $\bullet$ Abb. 3). Während Stefanie immer noch eine sehr ausgeprägte inflammatorische Komponente zeigte, war die Anzahl an Papeln und Pusteln bei Franziska unter der topischen Behandlung mit der Benzoylperoxid/Clindamycin-Kombination deutlich zurückgegangen.

Dadurch wuchs die psychische Belastung bei Stefanie derart an, dass auch sie auf Duac ${ }^{\circledR}$ Akne Gel $(1 \times$ täglich $)$ umgestellt werden musste. Wenige Wochen später hatte sich auch bei ihr das klinische Bild gebessert. .

Nach insgesamt 11 Wochen Behandlung mit dem gut verträglichen Kombinationspräparat wies Franziska kaum noch entzündliche Läsionen auf ( $\bullet$ Abb. 4), bei Stefanie war deren Anzahl nach nunmehr 8 Wochen deutlich zurückgegangen. Die letzten Bilder ( $\bullet$ Abb. 5) zeigen die Zwillingsschwestern 5 bzw. $5^{1} / 2$ Monate nach der Umstellung vom oralen Antibiotikum plus topischem Tretinoin/Erythromycin auf die ausschließlich lokale Kombinationsbehandlung mit Clindamycin/Benzoylperoxid. Zu diesem Zeitpunkt nahmen beide Schwestern nach Konsultation eines Gynäkologen seit 2 Monaten zusätzlich ein antiandrogen wirkendes Kontrazeptivum (Chlormadinonacetat/Ethinylestradiol). Die entzündlichen Effloreszenzen waren zu diesem Zeitpunkt nahezu vollständig abgeheilt und haben in unterschiedlichem Ausmaß Narben im Wangenbereich hinterlassen. Der Narbenbefund soll später durch Laserbehandlung und/oder Fruchtsäurepeeling kosmetisch korrigiert werden.

\section{Diskussion \\ $\nabla$}

3 Wochen nach der Therapieumstellung war bei den Schwestern ein deutlicher Unterschied im klinischen Befund festzustellen ( $\odot$ Abb. 1). Während Stefanies Akne immer noch eine sehr ausgeprägte inflammatorische Komponente zeigte, war die Anzahl an Papeln und Pusteln bei Franziska unter der topischen Behandlung mit der Benzoylperoxid/Clindamycin-Kombination deutlich zurückgegangen.

Die Tatsache, dass Franziska nach 3 Wochen alleiniger Duac ${ }^{\circledR}-$ Therapie eine deutliche Besserung gegenüber Stefanie zeigte, die die vorherige gemeinsame Therapie mit systemischem Clindamycin (morgens) und Tretinoin/Erythromycin Gel (abends) fortführte, legt den Schluss nahe, dass es sich bei Duac ${ }^{\circledR}$ Akne Gel um ein sehr wirksames Aknemittel handelt.

Im dargestellten „Doppel-Fall“ hat sich das topische Kombinationspräparat aus Clindamycin und Benzoylperoxid (Duac ${ }^{\circledR}$ Akne Gel) als zumindest ebenbürtige, wenn nicht bessere Alternative zur oralen Antibiotika-Therapie erwiesen, wobei sein Anwendungsgebiet auf die mittelschwere Akne begrenzt bleiben muss.

\section{Schlussbemerkung \\ $\nabla$}

Die Kompliziertheit des vorliegenden Falles zeigt, dass die Behandlung mittelschwerer bis schwerer Akne eine Herausforderung ist und in die Hand des Dermatologen gehört. Dabei sollte - z. B. über Zusatzleistungen - ein enger Kontakt zum Patienten gepflegt werden, um auf eventuelle Compliance-Probleme sofort reagieren zu können. Auch sollte im Bedarfsfall das volle therapeutische Spektrum ausgenutzt werden, um den Patienten vor der Entstehung bleibender, entstellender Narben zu bewahren. Der vorliegende Fall einer entzündlichen Akne bei Zwillingen hat demonstriert, dass ein neues, topisches Kombinationsarzneimittel bestehend aus $5 \%$ Benzoylperoxid und $1 \%$ Clindamycin 
durchaus die Wirksamkeit einer systemischen Antibiotika-Therapie erreichen oder gar übertreffen kann.

Schwere Akne hingegen ist und bleibt bei Vorliegen einer negativen Behandlungshistorie ein Fall für systemisches Isotretinoin.

\section{Abstract}

\section{Serious Acne Vulgaris in Monozygotic Twins - A Particular Challenge \\ $\nabla$}

After a series of less successful therapy efforts two 18 years old twin sisters presented themselves with a distinct acne papulopustulosa. Severity grade and localisation were very similar as expected within monovular twins. As the patients refused an oral isotretinoine therapy they firstly were treated with a systemic antibiotic in combination with a tretinoin/erythromycincream. Additional medical services ensured an intensive patient contact. Due to the unsatisfactory results one of the twin sisters changed to a new combination product containing $1 \%$ clindamycin and $5 \%$ benzoyl peroxyde (Duac ${ }^{\circledR}$ Acne Gel). Because of the visible improvements in this patient already after 3 weeks, the treatment of her sister was also changed towards this product exclusively. In both cases a significant reduction in the number of inflammatory lesions was observed, whereas the temporary delayed therapy change was obvious for a while. Compared with the treatment with a systemic antibiotic plus tretinoin/erythromycin cream the single treatment with Duac ${ }^{\circledR}$ Acne Gel finally proved to be more effective.

\section{Literatur}

1 Donald AE, Atkinson G, Langner A, Chu A, Goulden V, Clayton T. Efficacy and Safety of Duac ${ }^{\circledR} \mathrm{Gel}$ (a gel containing $1 \%$ clindamycin and $5 \%$ benzoyl peroxide) compared with Differin ${ }^{\circledR}$ Gel (a gel containing $0.1 \%$ adapalene) in the topical treatment of mild to moderate acne vulgaris (Abstract P01.139). London, UK 14th Cogress of the European Acadamy of Dermatology and Venereology; 12.-16.10.2005

2 Donald AE, Langner A, Sheehan-Dare R, Layton A. Efficacy of a new topical acne gel containing $1 \%$ clindamycin and $5 \%$ benzoyl peroxide compared with a solution containing $40 \mathrm{mg} / \mathrm{ml}$ erythromycin and $12 \mathrm{mg} / \mathrm{ml}$ zinc acetate (Abstract P130). New Orleans, USA 63rd Annual Meeting of the American Acadamy of Dermatology; 18. - 23. 02. 2005

3 Fagundes DS, Frazer JM, Klauda HC. New therapy update - A unique combination formulation in the treatment of inflammatory acne. Cutis 2003; 72 (Suppl 1): 16-19

4 Leyden JJ. Current issues in antimicrobial therapy for the treatment of acne. J Eur Acad Dermatol Venereol 2001; 15 (Suppl 3): 51 - 55

5 Lookingbill DP, Chalker DK, Lindholm JS, Katz HI, Kempers SE, Huerter CJ, Swinehart JM, Schelling DJ, Klauda HC. Treatment of acne with a combination clindamycin/benzoyl peroxide gel compared with clindamycin gel, benzoyl peroxide gel and vehicle: combined results of two double-blind investigations. J Am Acad Dermatol 1997; 37: 590 - 595

6 Ozolins M, Eady EA, Avery AJ, Cunliffe WJ, Po AL, O'Neill C, Simpson NB, Walters CE, Carnegie E, Lewis JB, Dada J, Haynes M, Williams K, Williams HC. Comparison of five antimicrobial regimens for treatment of mild to moderate inflammatory facial acne vulgaris in the community: randomised controlled trial. 2004; 364: 2188 - 2195

7 Walton S, Wyatt EH, Cunliff WJ. Genetic control of sebum excretion and acne - a twin study. Br J Dermatol 1989; 121: 144- 145

\section{Buchbesprechung}

Hautkrebs - Ein oft unterschätztes Risiko.

Risikofaktoren, Diagnostik, Therapie und Prognose. Rat \& Hilfe P. Altmeyer, S. Reich

Stuttgart: Kohlhammer, 2006. 184 S., Kart. 19,80€

ISBN 3-17-018620-5

Hautkrebse sind häufig und vielgestalt, sie sind offensichtlich einsehbar und erkennbar und dennoch sind sie noch oft tabubehaftet und werden verniedlicht. Und dies, obschon die hauptsächliche Ursache, übermäßige Lichtbelastung der Haut, bekannt ist. Es ähnelt die Geschichte in vielem derjenigen der Krebse an Lippen, im Hals und in den Lungen in Beziehungen und Abhängigkeit vom Rauchen. Nur kann man die Hautkrebse viel besser und früher erkennen, und beide, Hautkrebse und Raucherkrebse, könnte man ja verhindern, wenn man nur wollte und dazu die präventive Kraft aufbrächte. Denn neben der Klärung der Entstehungsweise und der Früherkennung ist das probate Mittel in jedem Fall die primäre Prävention, die Vermeidung durch Weglassen des Rauchens im einen Fall, und durch vernünftiges Sonnenverhalten im Fall der Hautkrebse.

Solches zu fördern, bedarf es vielfältiger und flächendeckender Bemühungen, Aufklärung, Information, Hilfe und Überzeugung. Nicht zuletzt durch Repetition! Dies alles soll fundiert, eindrücklich und fachmännisch geschehen und wirkungsvoll sein. Ein Mittel dazu sind gute und eingängige Schriften, wie sie der Verlag W. Kohlhammer herausgibt, 14 bis jetzt zum Thema Krebs und $11 \mathrm{zu}$ anderen medizinischen Themen. Und diejenige zum Hautkrebs liegt nun vor aus der Bochumer Dermatologie, die sich zu diesem Thema in Fachkreisen und patientenorientiert, in Forschung und in der Lehre verdient gemacht hat.

Es ist ein gelungenes Werk mit straffem Text, guten Bildern und bester Gliederung. Auf 184 Seiten sind alle Hautkrebse dargestellt, die häufigen Basaliome, Melanome, Spinaliome und Lymphome, die eher seltenen Merkelzell-Tumoren, die bindegewebigen Tumoren, die Präkanzerosen und sogar die paraneoplastischen Erscheinungen. Man erhält Antworten und Informationen zu allen gängigen Fragen und man findet diese auch sehr gut. Die Gliederung hilft dabei, das Inhaltsverzeichnis und ein Stichwortregister. Das Glossar wirkt bei Begriffen und Fremdwörtern trefflich.

Ein kleines, handliches und hervorragendes Büchlein, das weite Verbreitung verdient und enorm hilfreich ist.

E. G. Jung, Heidelberg 